\title{
Path Stability Mechanism in Mobility based AOMDV for MANETS
}

\author{
Vishesh D. Savane \\ P. G. Dept, \\ MBES College of Engineering, \\ Ambajogai, India, 431517.
}

\author{
Veeresh G. Kasabegoudar \\ P. G. Dept, \\ MBES College of Engineering, \\ Ambajogai, India, 431517.
}

\begin{abstract}
Mobile Ad hoc Networks (MANETs) are used for providing Quality of Service (QoS) where nodes are having mobility and can travel in any random direction. There are several reactive protocols (DSDV, AODV, EBAODV, etc.) available in literatures which are suitable for MANETs based on single and multiple paths. Among these, AOMDV protocol is the most suitable for finding multiple paths to the destination. However, in its basic version does not consider mobility of intermediate nodes which causes packet loss in traveling. In this work to enhance the path stability, mobility of an intermediate node and exclude the selection of high mobility node is introduced. Mobility of nodes is calculated by using RREQ packet retransmission strategy. The intermediate results are stored in routing table and this information is forwarded to source node for path selection process. Finally, intermediate node having least mobility is selected by the source for data packet transmission towards destination. For this we used AOMDV protocol with three different mobility models (Random way point, Random Walk, and Gauss Markov). All the models are investigated using network simulator and their comparisons are presented and found satisfactory.
\end{abstract}

\section{General Terms}

Mobile Ad hoc Networks, AOMDV, Routing.

\section{Keywords}

Mobility Model, Path Stability, Reactive Protocols.

\section{INTRODUCTION}

A MANET is a self-organizing collection of wireless mobile nodes that form a temporary and dynamic wireless network established by a group of mobile nodes on a shared wireless channel without a fixed networking infrastructure or centralized administration [1]. Mobile nodes are connected wirelessly to each other by radio links for limited range. In MANET's QoS is required for effective communication and best effort applications such as file transfer and so on. However, QoS provisioning in MANET is a very challenging task. The main reasons behind this are being unpredictable nodes mobility, limited battery power, and range of mobile devices [1].

Due to free movement of the mobile nodes in Adhoc network, maintaining network state and topology is very difficult task thus achieving path stability for data transmission is difficult [2]. Number of routing protocols is available in literature which subject to solve such problems. If path gets failed while data packet transmission, source node repairs path or restarts the route selection procedure towards destination which results in control packet overhead in network and leads bandwidth wastage [2]. Along with this many other challenging factors and problems such as Chanel Capacity Constraints, Unpredictable Channel Access Delay, Inaccurate Bandwidth Estimation, and Network Heterogeneity are available for providing QOS in MANET [3-9]. To address such challenges number of design philosophy and routing protocols are available. Based on how the information is acquired and maintained, MANET's routing protocols are categorized in to proactive, reactive, and hybrid [3]. Brief information about these categories is given in the following paragraphs.

The main characteristic of proactive routing is that it maintains the constant routing path to each node to each other node in routing table which is stored at every node. The route creation and maintenance is done by using periodic or event driven updates by using packet. The source node can get a routing path immediately from routing table if it needs for data transmission to desired destination [3].

Reactive protocols are also called "on-demand" protocols. These on demand protocols do not require the maintenance of routing table at every node as done in proactive when there is no traffic. In reactive protocols whenever a traffic source node needs a route, it initiates a route discovery process by sending a route request packet towards destination and waits for a route reply packets from neighboring nodes. However, route maintenance in routing table is an important operation of reactive routing protocols, because source nodes may suffer from long delays for route searching due to mobility of node [3].

A hybrid protocol as the name indicates it is a combination of both proactive and reactive strategies. Route creation and maintenance is done using both proactive and reactive methods. Hence, hybrid protocols address both efficiency and robustness $[3,6]$.

In this work the mobility based AOMDV protocol for stable path to the destination is proposed. Here, the mobility of node can be achieved by recording number of times the RREQ packets are retransmitted to the next neighboring node. Based on this calculation, forward path is selected for data packets transmission. Lowest mobility value of neighboring intermediate node is considered by source node for achieving the stabilized path. Also, we compare this approach in three different mobility models namely; Random way point, Random Walk and Gauss-Markov mobility model and performance is analyzed in each one of these techniques. The reason to for different mobility models is that mobility models are designed to describe the movement pattern of mobile 
users, and how their location, velocity and acceleration change over time $[5,8]$.

In Section 2, related work on the AOMDV and other reactive protocols is presented. Section 3 explains the objective of the proposed work. Simulation results and discussions of the study are presented in Section 4 followed by conclusions of this work in Section 5.

\section{RELATED WORK}

There are several works reported in literature on various reactive protocols, comparisons, and their variants [3-15]. Therefore, this work primarily focuses on reactive protocols as here routes are acquired when there is data traffic on source for transmission and path search done on requirement. Among the reactive protocols, Adhoc on Demand Distance Vector (AODV) [2] and Adhoc on Demand Multipath Distance Vector (AOMDV) [4] are mostly used for single path and multipath respectively.

AODV protocol has attracted great attention because of its characteristics, like simplicity in nature, low computational complexity and low processing overhead and so on [10]. It is an on demand routing protocol, so that a route is only discovered when required by a source node. This eliminates periodic routing updates and only necessary information is propagated to minimize control overhead [3]. In AODV, each node maintains a routing table to record routing information obtained from routing packets. Whenever a traffic source needs a route, it initiates a route discovery process by sending a route request for the destination and waits for a route reply [8].

AOMDV extends the AODV protocol to discover multiple paths between the source and the destination in every route discovery process. Multiple paths so computed are guaranteed to be loop-free [4]. In the route discovery process of AOMDV, the RREQ packets are flooded network-wide so that a node may receive several copies of the same RREQ. All duplicate copies that arrive later are examined for finding alternate multiple forward and reverse paths. All the discovered paths are listed and stored in the routing table. But reverse paths and forward path are formed by only those copies that preserve the loop-free path. AOMDV computes alternate paths with minimal additional overhead over AODV; it does this by exploiting already available alternate path routing information [7].

Channel-Aware AOMDV (CA-AOMDV) [14] uses the channel average non fading duration as a routing strategy to select stable links for path discovery, and applies a preemptive handoff strategy to maintain reliable connections by exploiting channel state information. Using the same information, paths can be reused when they become available again, rather than being discarded. Author provides new theoretical results for the downtime and lifetime of a live-dielive multiple path system

Ad hoc on-demand trusted multi-path distance vector routing (AOTMDV) protocol based on trust prediction model is proposed for MANETs [15]. This protocol provides a flexible and feasible approach to choose the shortest path that meets the security requirements of data packets transmission along with malicious node detection in multi-agent systems and threat mitigation from all available nodes. In this protocol, a source can establish multiple loop-free trusted paths to a destination in one route discovery process without mobility information.
The present AOMDV doesn't reflect any kind of mobility information of the nodes. Therefore, the possibility of the path break may be high because it chooses the path which includes the nodes with high mobility and highest sequence number. If path based on mobility of intermediate nodes is selected and excluding the selection of high mobility node results in the path stability and performance of protocol $[6,14,15]$.

Mobility models mimic the movements of real mobile node [5]. Changes in speed and direction must occur in reasonable time slots. In order to thoroughly simulate a new protocol for an ad hoc network, it is important to use mobility model that accurately represents the mobile nodes that will move and eventually utilize the given protocol correctly. Only in this type of mobility models it is possible to determine whether or not the proposed protocol will be useful when implemented [5].For this purpose the proposed concept is introduced in the following three different mobility models.

\subsection{Random Waypoint Mobility Model}

The Random Waypoint Mobility Model includes pause times between changes in direction and/or speed. A mobile node begins for movement by pausing in one location for a certain amount of time after pause time expires, the mobile node chooses a random destination address and speed in the simulation area, and Speed is uniformly distributed between max and min. The mobile node then travels toward the newly chosen destination at the selected speed and this process continues until simulation time expires [5].

\subsection{Random Walk Mobility Model}

The Random Walk Mobility Model was first described mathematically by Einstein in 1926 [5]. Many objects like human and mobile nodes in real world move in extremely unpredictable ways, the Random Walk Mobility Model was developed to mimic this situation near to this. In RWMM, mobile node moves from its current location to a new location by randomly choosing a direction and speed. The new selected speed and direction are both selected from predefined ranges, $(\min , \max )$ and $(0, \pi)$ respectively. Movements of mobile nodes occur in either a constant time interval $t$ or a constant distance traveled $d$, at the end of a new direction and speed are calculated [5].

\subsection{Gauss-Markov Mobility Model}

The Gauss-Markov Mobility Model was designed to adapt for different levels of randomness via one tuning parameter. Initially mobile node is assigned a current speed and direction. At fixed intervals of time, $n$, movement occurs by updating the speed and direction of each mobile node. Specifically, the value of speed and direction at the $n^{\text {th }}$ instance is calculated based upon the value of speed and direction at $(n-1)^{s t}$ instance and a random variable using the following equations [5]:

$$
\begin{aligned}
& s_{n}=\alpha s_{n-1}+(1-\alpha) \bar{s}+\sqrt{\left(1+\alpha^{2}\right)} s_{x_{n-1}} \\
& \left.d_{n}=\alpha d_{n-1}+(1-\alpha) \bar{d}+\sqrt{\left(1+\alpha^{2}\right.}\right) d_{x_{n-1}}
\end{aligned}
$$

Where $s_{n}$ and $d_{n}$ are the new speed and direction of a mobile node at time interval $n$; where $\alpha$ with value $0<\alpha<1$, is the tuning parameter used to vary the randomness; $s$ and $d$ are constants representing the mean value of speed and direction as $n \rightarrow \infty$; and $s_{x_{n-1}}$ and $d_{x_{n-1}}$ are random variables from a Gaussian distribution Totally random values (or Brownian 
motion) are obtained by setting $\alpha=0$ and linear motion is obtained by setting $\alpha=1$. In each time interval the next location is calculated based on the current location, speed, and direction of movement. Specifically, at time interval $n$, a mobile node position is given by the equations [5]:

$$
\begin{aligned}
& x_{n}=x_{n-1}+s_{n-1} \cos d_{n-1} \\
& y_{n}=y_{n-1}+s_{n-1} \sin d_{n-1}
\end{aligned}
$$

Where $\left(s_{n}, y_{n}\right)$ and $\left(s_{n-1}, y_{n-1}\right)$ are the $x$ and $y$ coordinates of the mobile nodes position at the $n^{\text {th }}$ and $(n-1)^{s t}$ time intervals, respectively, and $s_{n-1}$ and $d_{n-1}$ are the speed and direction of the mobile node, respectively, at the $(n-1)^{s t}$ time interval.

\section{ROUTE SELECTION PROCEDURE IN AOMDV WITH MOBILE NODES}

AOMDV [4] finds routes on demand using a route discovery procedure. Here, the objective is to find path in a route discovery process based on mobility of mobile nodes as suggested in [6]. Each one of the mobility models is studied with AOMDV using Network Simulator (NS2) software and results are presented in Section 4.

\subsection{Route Discovery}

In AOMDV protocol the source node propagates RREQ packets towards the destination node through intermediate nodes to establish multiple loop free paths [4]. If next node is not in range of source node, RREQ packet retransmission counter is increased [6-7]. In proposed work as shown in figure 1 the algorithm for route discovery process is used to update mobility value of next neighboring mobile node. This process is also used by all intermediate nodes and consecutively updated mobility values are stored in routing table. For storing values mobility field is added in to routing table.

If (request retransmission counter is greater than 0) then Increase mobility value in the routing table by one, and, send RREP packet to source node with mobility value of routing node

Figure 1: Algorithm for route discovery process at source node and intermediate node.

\subsection{Data Packet Forwarding and Route \\ Maintenance}

The AOMDV [4] protocol uses fairly reliable unicast RREPs packet for data packet forwarding path selection. The source node uses forward path for data traffic transmission based on highest sequence number from received RREP packets. In proposed work, as shown in Figure 2 if RREP packets are received at the source node, it checks mobility information present in RREP packets rather than sequence number. In this source node searches least mobility value from all received RREP packets and generate forward path by using source of RREP packet.

If (smaller mobility value in RREP) then

Generate forward path via source of RREP packet

Figure 2: Algorithm for route selection process at source node.

Intermediate nodes use different criteria as shown in Figure 3. Before forwarding RREP packet, it compares the mobility value present in RREP packet with its own routing table mobility value. And, if its own value is greater than received mobility value then RREP packet mobility value is updated otherwise it will be forwarded as it is. To achieve this extra field is added in RREP.

$$
\begin{aligned}
& \text { If (mobility value of routing table is less than mobility } \\
& \text { value in RREP packet) then } \\
& \text { Forward RREP packet as it is to source node } \\
& \text { Else } \\
& \text { Update RREP packet mobility value by routing table } \\
& \text { value }
\end{aligned}
$$

Figure 3: Algorithm for RREP packet forwarding process at intermediate node.

For route maintenance if HELLO packet is received at the node then mobility value is decreased by one. In the proposed work, source selects the path with reflecting the mobility information of the nodes. This lowers the possibility of the route break. Here the route is obtained which is more stable than the existing AOMDV. By using the mobility information of the node the total data throughput and performance is improved [6].

\section{SIMULATION RESULTS AND DISCUSSIONS}

The performance of the AOMDV is investigated with three different mobility models in NS-2. Among three the Random Way Point Model has been studied with NS2. However, the remaining two models i.e., Random Walk Model and GaussMarkov models are not supported by NS2, these are investigated with the help of BONNMOTION [16] simulator. Here, totally 40 nodes are randomly arranged besides five source nodes which are randomly selected for transmission of four packets per second with queue size of 150 packets along with the following parameter as shown in Table 1.

In Gauss-Markov mobility model angle of standard deviation and speed of standard deviation is kept 5 and 20 respectively. For the study three performance parameters which are throughput, reply packets overhead, and control packets overhead against time parameter are taken. Simulation results of all these performance parameters are presented in the following paragraphs. Performance of the proposed work (AOMDV with different mobility models) is compared in three different mobility models as mentioned above.

Table 1: Simulation environment

\begin{tabular}{|c|c|c|c|}
\hline Parameter & $\begin{array}{c}\text { Random } \\
\text { Way point }\end{array}$ & $\begin{array}{c}\text { Random } \\
\text { Walk }\end{array}$ & $\begin{array}{c}\text { Gauss- } \\
\text { Markov }\end{array}$ \\
\hline $\begin{array}{c}\text { Simulation } \\
\text { area }\end{array}$ & $\begin{array}{c}1100 \mathrm{~m} * \\
1100 \mathrm{~m}\end{array}$ & $\begin{array}{c}1100 \mathrm{~m} * \\
1100 \mathrm{~m}\end{array}$ & $\begin{array}{c}1100 \mathrm{~m} * \\
1100 \mathrm{~m}\end{array}$ \\
\hline $\begin{array}{c}\text { Simulation } \\
\text { time }\end{array}$ & $500 \mathrm{sec}$. & $500 \mathrm{sec}$. & $500 \mathrm{sec}$. \\
\hline $\begin{array}{c}\text { Node speed } \\
\mathrm{m} / \mathrm{sec} .\end{array}$ & $\begin{array}{c}1 \sim 25 \\
\mathrm{~m} / \mathrm{sec} .\end{array}$ & $\begin{array}{c}1 \sim 25 \\
\mathrm{~m} / \mathrm{sec} .\end{array}$ \\
\hline Pause time & $5 \mathrm{sec}$. & $5 \mathrm{sec}$. & $5 \mathrm{sec}$. \\
\hline $\begin{array}{c}\text { Traffic } \\
\text { pattern }\end{array}$ & $\mathrm{CBR}$ & $\mathrm{CBR}$ & $\mathrm{CBR}$ \\
\hline
\end{tabular}

The throughput is recorded as the number of data packets transmitted from the source node to the destination node. Figure 4(a) shows packet throughput over time of AOMDV protocol in three different mobility models. 
Figure 4(b) shows packet throughput over time of the proposed AOMDV protocol in three different mobility models and results show throughput increases in all three mobility models compare to AOMDV protocol due to more stabilized path search towards destination node. Also, among the different mobility models, Random Way Point Model yields better throughput than other models when the time progresses.

The simulation results of other two performance parameters (reply packets and control packets overhead) are shown in Figures 5 (a) \& (b), and 6 (a) \& (b). From these two figures it may be noted that AOMDV with mobility performance is better than conventional AOMDV protocol performance. As stated earlier, it may also be noted that among the three mobility models.

Random Way Point model shows the better overall performance than other two mobility models. The reply packets required is least for the case of AOMDV with Random Way Point model (Figure 5 (b)). Similarly, least control packets overhead can be seen in AOMDV with GaussMarkov model (Figure 6(b)).

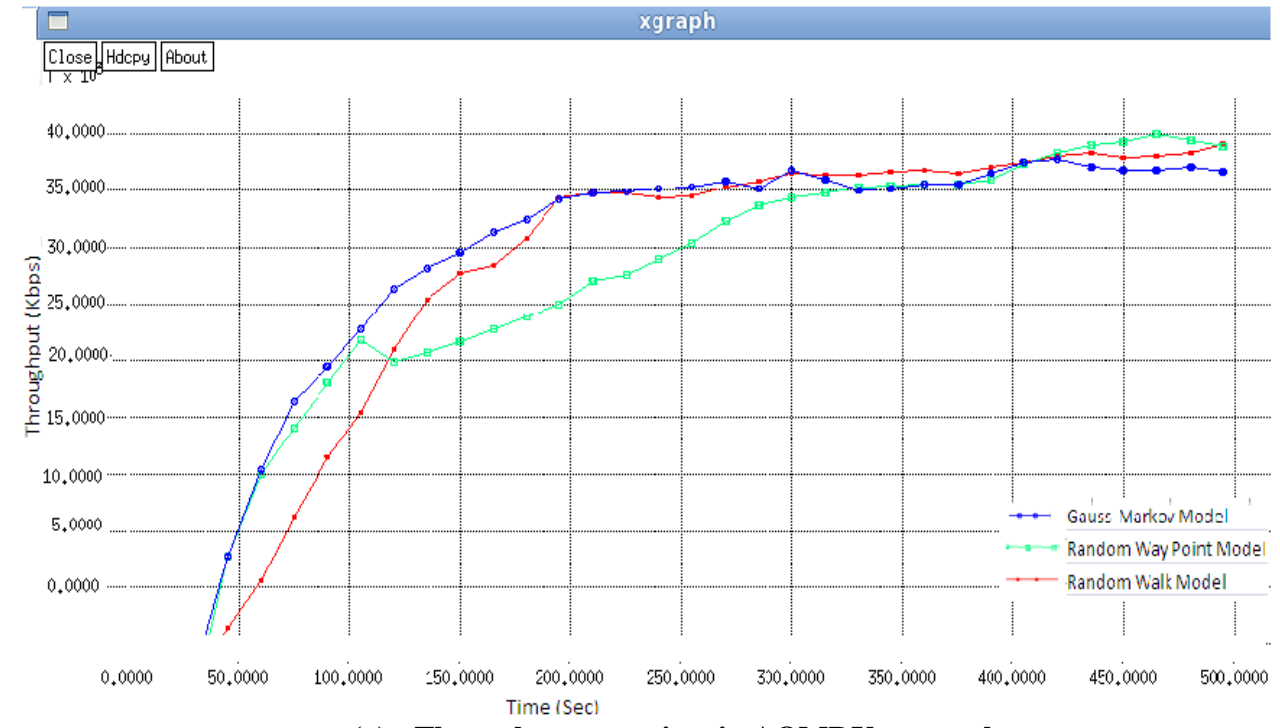

(a) Throughput over time in AOMDV protocol.

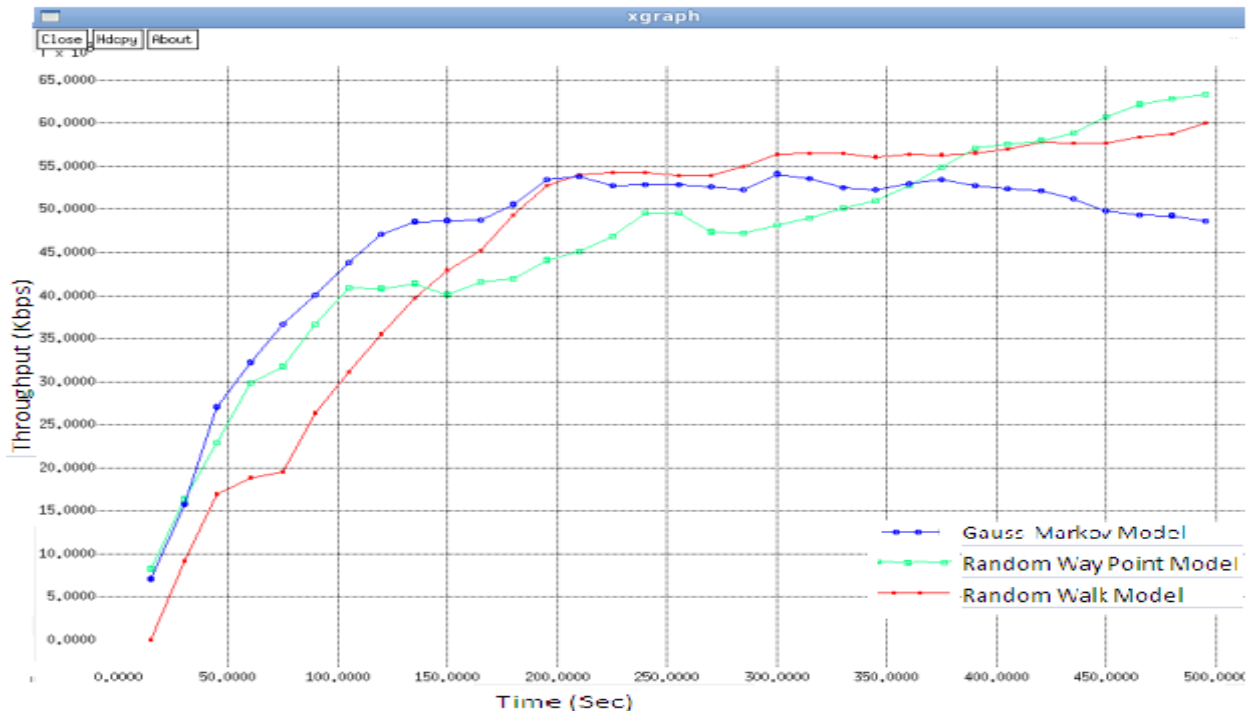

(b) Throughput over time in proposed AOMDV with different mobility models.

Figure 4: Throughput over time in conventional and proposed AOMDV protocols. 


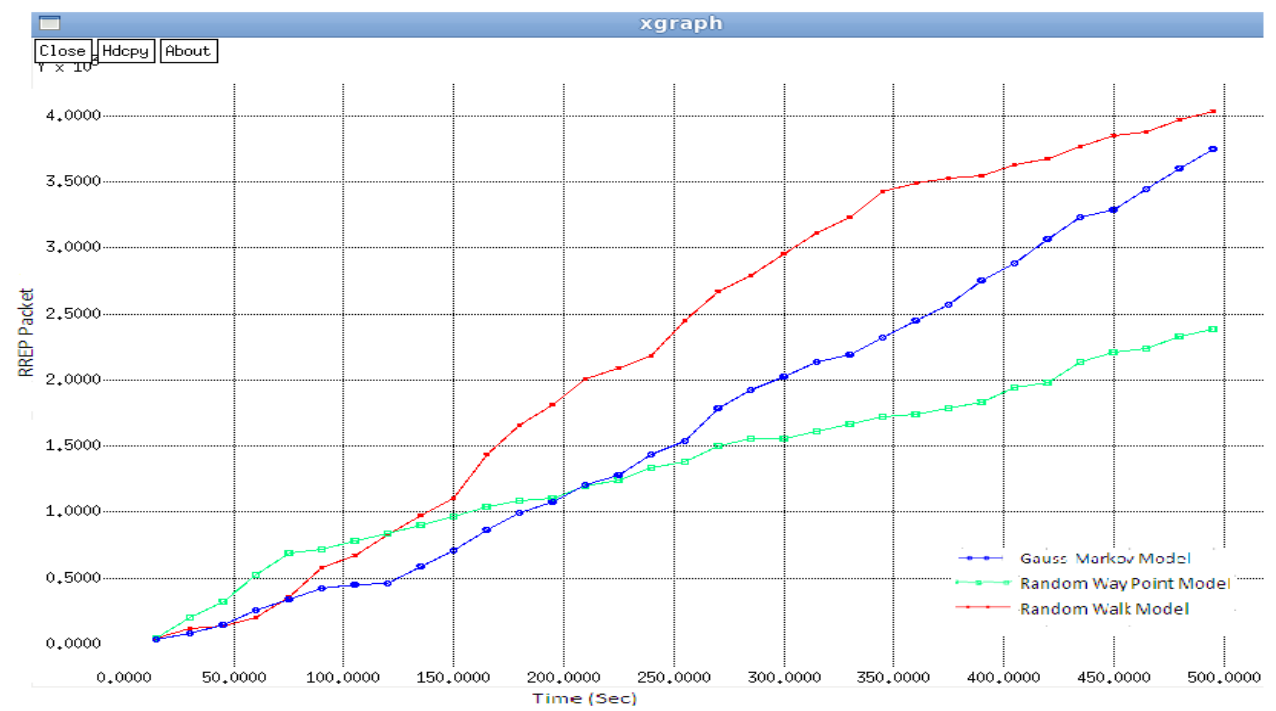

(a) RREP packets in basic AOMDV protocol.

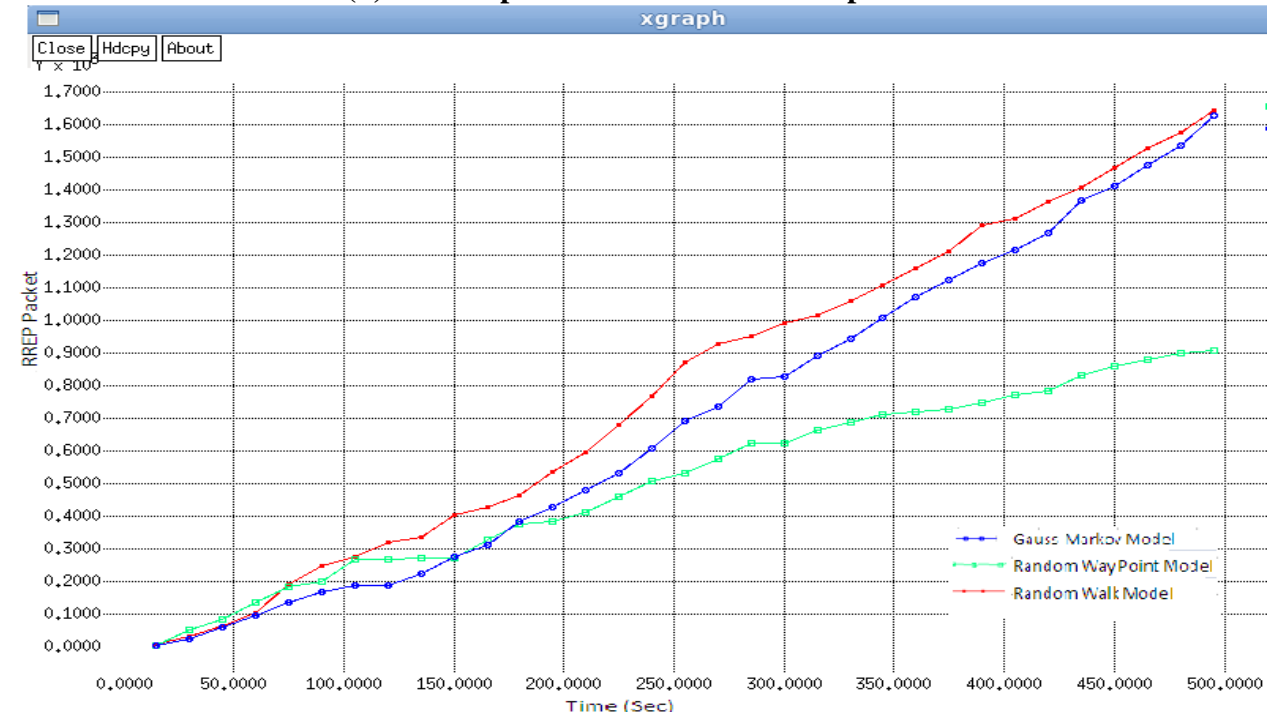

(b) RREP packets in proposed AOMDV protocol with different mobility models.

Figure 5: RREP packets over time in proposed AOMDV with different mobility models.

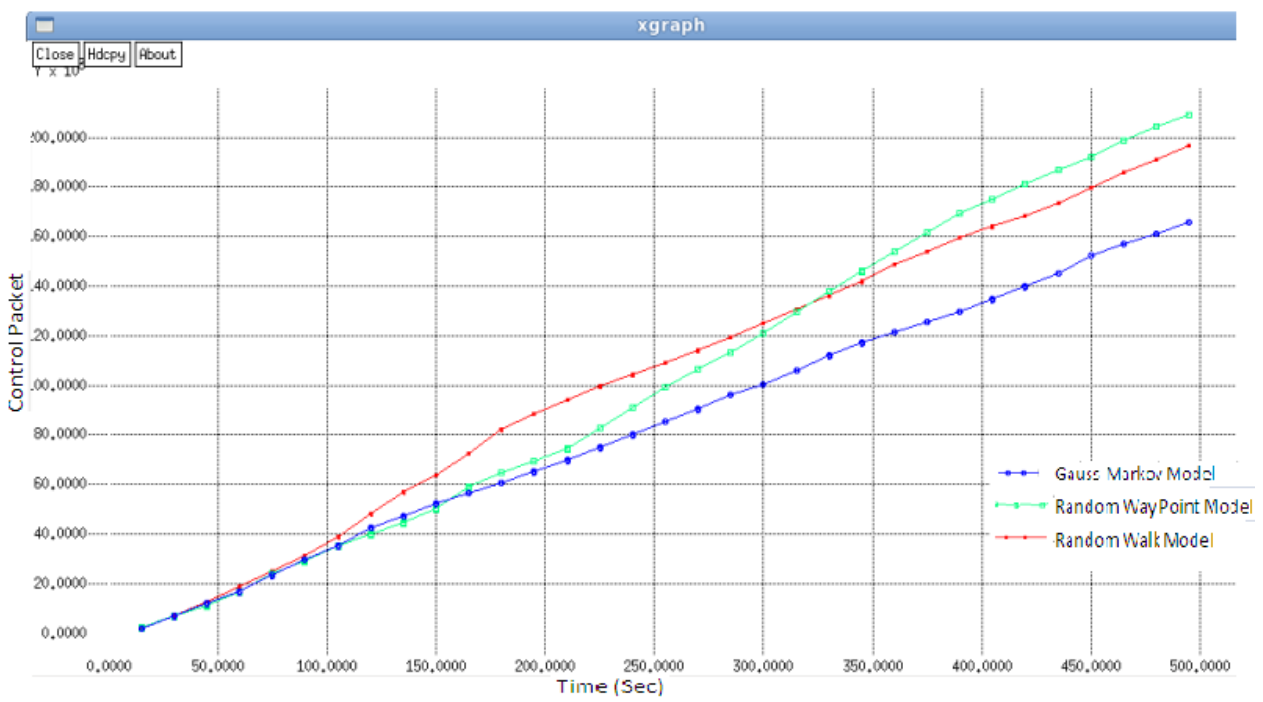

(a) Control packets in basic AOMDV protocol. 


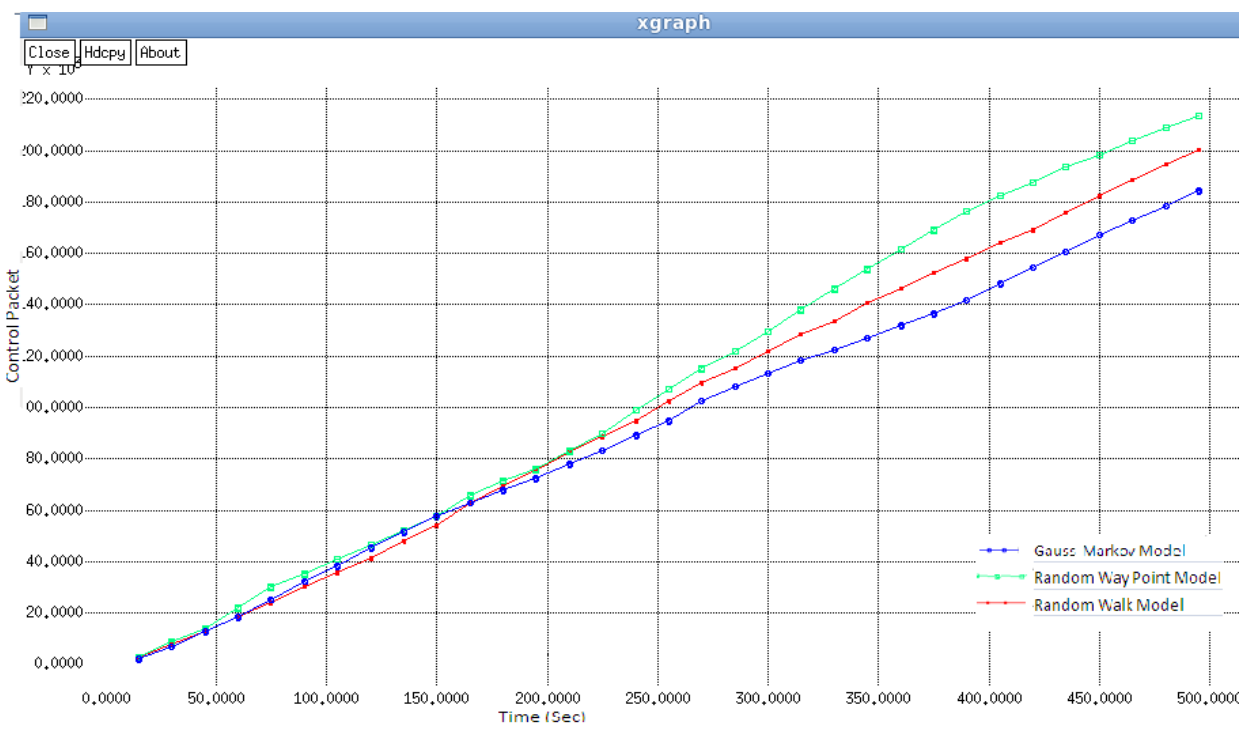

(b) Control packets in proposed AOMDV protocol with different mobility models.

Figure 6: Control packets over time in proposed AOMDV with different mobility models.

\section{CONCLUSIONS \& FUTURE WORK}

Location finders like GPS devices are used for finding the location and mobility of mobile nodes but in the proposed work it is demonstrated that how the mobility of mobile nodes is identified. The identification of mobility of mobile nodes can be tackled by retransmission strategy. When the request from source node does not reach the next node then request is retransmitted. This implies that the node is not in the range of source node and having high mobility. Hence, such nodes are discarded from the route selection. From the simulation results presented here it may be observed that among the three mobility models considered Random Way Point Mobility Model helps in the selection of more stable path. The future work of this investigation includes the improvement of overall performance (throughout and control packets overhead) further for which it is planned to introduce timeout/delay counter to reduce the packets dropping rate.

\section{REFERENCES}

[1] I. Chlamtac A, Marco Conti B, Jennifer J.-N. Liu, "Mobile ad hoc networking: imperatives and challenges," Ad Hoc Networks, pp. 13-64, 2003.

[2] S. Sesay, Zongkai Yang and Jianhua He, "A survey on mobile Adhoc wireless networks," Information Technology Journal, pp. 168-175, 2003.

[3] S. Sridhar and R. Baskaran, "A survey on QoS based routing protocols for MANET," International Journal of Computer Applications, vol. 8, no. 3, pp. 15-22, 2010.

[4] M. K. Marina and S. R. Das, "Ad hoc on-demand multipath distance vector routing," Wireless Communications And Mobile Computing, pp. 969-988, 2006.

[5] T. Camp, Jeff Boleng and Vanessa Davies, "A survey of mobility models for ad hoc network research," Wireless Communication \& Mobile Computing, vol. 2, no. 5, pp. 483-502, 2002.

[6] R. Park, Wuwoan Kim, Sangdong Jang, "Improved AOMDV to increase path stability by utilizing the node mobility information in MANET," IEEE-ICOIN, pp. 417-422, 2013

[7] F. Zi Fu, Z. Ling and W. X. Yu, "An optimized AOMDV routing algorithm based on self-adaptive node speed in wireless sensor network," IWIEE, pp. 33-37, 2012.

[8] Z. Yan, Wu Mu-qing, Wu Da-peng, and Xu Chun-xiu, "Toward path reliability by using adaptive multi-path routing mechanism for multimedia service in mobile Adhoc network," The Journal of China Universities of Posts and Telecommunications, pp. 93-100, 2010.

[9] H Babaei and M. Romoozi, "Multi objective AODV based on a realistic mobility model," IJCSI, vol. 7, no 3, pp. 8-6, 2010.

[10] A. Ambhaikar, H.R. Sharma and V. K. Mohabey, "Improved AODV protocol for solving link failure in MANET," IJSER, vol. 3, no.10, pp. 1-6, 2012.

[11] D. Cerri and Alessandro Ghioni, "Securing AODV: The A-SAODV secure routing prototype," IEEE Communications Magazine, pp. 120-125, 2008.

[12] K.W. Wong, H. Liu, X. Chu and Y. W. Leung, "Efficient broadcasting in multi-hop wireless networks with a realistic physical layer," Elsevier Adhoc Networks, no. 11, pp. 1305-1318, 2013.

[13] R. Bai and Mukesh Singhal, "DOA: DSR over AODV routing for mobile adhoc networks," IEEE Transactions on Mobile Computing, vol. 5, no. 10, pp. 1403-1416, 2006.

[14] X. Chen, Haley M. Jones, and Dhammika Jayalath, "Channel-Aware routing in manets with route handoff," IEEE Transactions On Mobile Computing, vol. 10, no. 1, pp. 108-121, 2011.

[15] H. Xia, Z. Jia, L. Ju, X. Li, and Edwin HM, "Impact of trust model on on-demand multi-path routing in mobile ad hoc networks," Elsevier Computer Communications, no. 36, pp. 1078-1093, 2013.

[16] The BonnMotion Software available online at: (http://www.sys.cs.uos.de/bonnmotion/). 